\title{
Pair production in thermal plasmas: a computer model
}

\author{
Susan Stepney Institute of Astronomy, Cambridge
}

\begin{abstract}
A computer code has been developed to follow the processes of electronpositron pair production, annihilation, bremsstrahlung and Comptonization in a slab of mildly relativistic thermal plasma. The resulting equilibrium solutions are compared with the semi-analytic calculations of Svensson[1] .
\end{abstract}

\section{Introduction}

The various processes occurring in relativistic plasmas have been discussed elsewhere[2]. Since the electron thermalization timescale[3] is less than the bremsstrahlung cooling timescale for $k T \lesssim 10 m_{e} c^{2}$, it is self-consistent to consider thermal plasmas at mildly relativistic temperatures. In this paper I discuss a computer code that has been developed to model slabs of thermal plasma at temperatures of $k T \lesssim m_{e} c^{2}$ and various optical depths.

The starting point is a one-D radiative transfer code, written by Guilbert[4], to study the Compton cooling of hot gas by an external source of soft photons. It correctly treats Comptonization at mildly relativistic temperatures. I have extended the program to include internal sources and sinks of photons, and pair production.

The processes considered are:

(1) Comptonization

(2) Thermal Bremsstrahlung:

(i) electron-proton $(e-p)$ : This is very straight-forward to calculate, being merely a single integral over the cross section.

(ii) electron-electron $(e \pm e \pm)$ : This emission is comparable to (i) at temperatures $\gtrsim$ $50 \mathrm{keV}$, but the calculation of the thermal spectrum requires the evaluation of a 5-D integral. I use the fit recently calculated by Guilbert[5].

(iii) electron-positron: The spectrum is still unknown at mildly relativistic temperatures. I estimate its contribution by putting it equal to the $e-p$ rate at low temperatures (since we are interested in photons with energies $\gtrsim 0.5 \mathrm{Me} \mathrm{V}$, one of the radiating electrons will be relativistic, as in the case of the high energy tail of $e-p$ bremsstrahlung), twice the $e-e$ rate at high temperatures (the ultra-relativistic limit), and joining smoothly in between. Since the equilibrium pair densities are low, the error made by using this approximation is small.

(3) Pair production: The dominant production mechanism, and that which takes most of the computing time, is that of photon-photon collisions. Photon-electron collisions account for $10-20 \%$ of the pair production. The other processes (photon-proton and particle-particle) are included for completeness, since they take a negligible amount of computing time.

\section{The Model}

Equilibrium models are characterized by two parameters: the temperature and an optical depth. Since the pair density is the quantity to be determined, the electron scattering depth is not a suitable parameter to chose. Instead the "proton optical depth" $\tau_{p}=N_{p} \sigma_{T} R$ is used. $\tau_{p}$ is related to the Thomson optical depth $\tau_{e s}$ by $\tau_{e s}=(1+2 z) \tau_{p}$, where $z$ is the number of electron-positron pairs per proton.

I have run models for various combinations of $\mathrm{T}$ and $\tau_{p}$. Initially the slab has no photons or pairs present. At each timestep the photon and pair densities are incremented as required, and the photons are Comptonized and transported spatially. The pairs are assumed to stay

Susan Stepney. Pair production in thermal plasmas: a computer model.

In M. L. Burns, A. K. Harding, and R. Ramaty, editors, Positron-Electron Pairs in Astrophysics,

AIP Conference Proceedings, 101, 1983. 
where they are produced, to be instantly thermalized and to have an isotropic distribution. The model is then allowed to evolve either to an equilibrium solution, or until the pair density diverges.

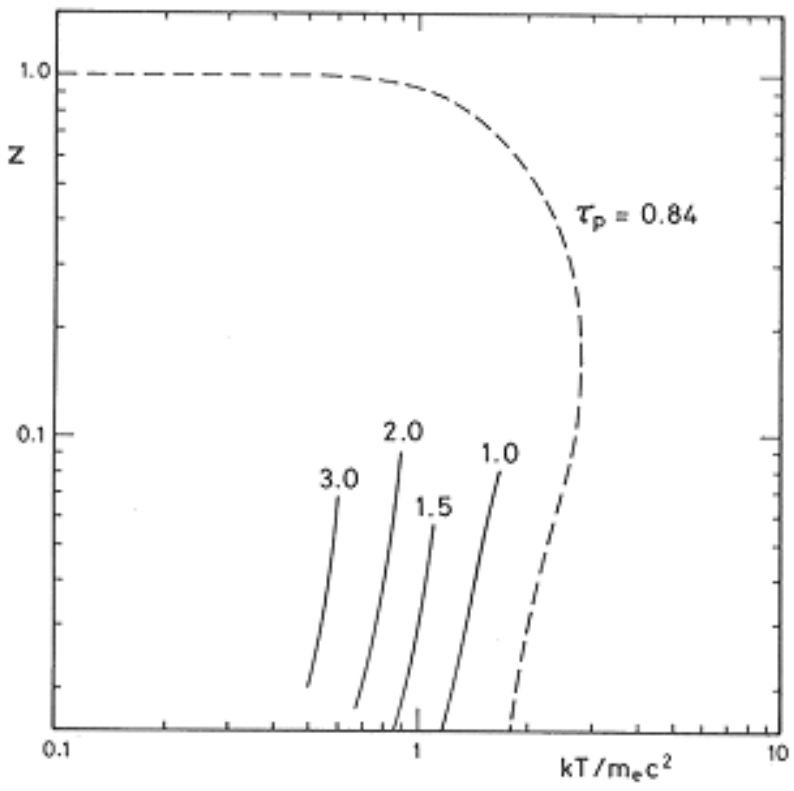

Figure 1. Equilibrium pair density as a function of temperature. The curves are labelled by $\tau_{p}=N_{p} \sigma_{T} R$. The doted curve is Svensson's result for $\tau_{p}=0.84$.

It is well known that there are some combinations of temperature and optical depth for which no equilibrium solution exists. This is due to the fact that the annihilation cross section decreases at high energies, but that the pair production rate increases. At high enough temperatures the pairs are created faster than they can be destroyed. Physically, any extra energy goes into increasing the total number of particles, rather than their mean energy. The maximum temperature, in the limit of zero optical depth, is $k T \simeq 24 m_{e} c^{2}$.

\section{Results}

The results of these runs are shown in the figures, along with Svensson's[1] semi-analytical results for comparison. Svensson calculates the equilibrium solutions by assuming isotropic, homogeneous particle and photon distributions, and neglecting Comptonization. For each value of temperature and optical depth there are either two or no solutions for the equilibrium pair density, z. In the region where there are two solutions the higher one represents an unstable equilibrium - the plasma has negative specific heat. This solution is not found by the computer program.

In figure 1 it can be seen that, for a fixed proton optical depth, the pair density increases rapidly with temperature. The slopes of the lines are consistent with Svensson's low pair density solutions. The solid line in figure 2 shows the boundary between models which had equilibrium solutions and those which diverged. The slope of this line is also consistent with the envelope of the semi-analytic constant $z$ models. It is, however, somewhat lower. This is the result of including Comptonization and inhomogeneous distributions. Since the pair and photon densities are higher at the centre of the slab, this mimics a higher optical depth (or temperature) and so the runaway occurs sooner. In fact, the two methods are complementary. Svensson's results can be found relatively quickly, and also the method finds the high pair density branch, but it is only valid for small optical depths. The model discussed here requires much more computing time, but is valid for optical depths greater than unity. 


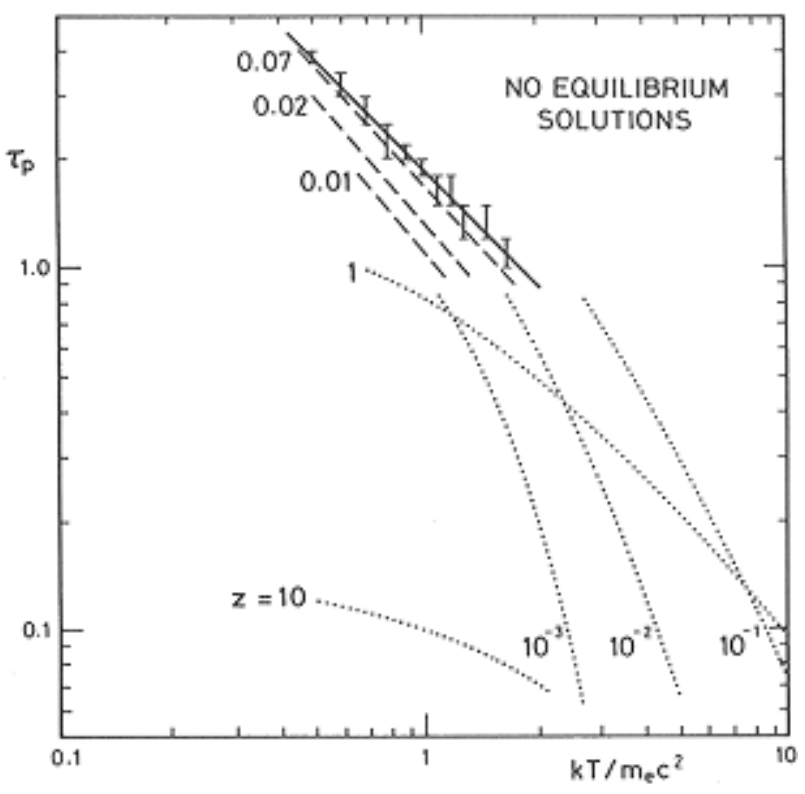

Figure 2. Proton optical depth as a function of temperature. The curves are labelled by $z$. The bottom of an error bar represents a model which converged, the top, one which diverged. The dotted curves are Svensson's results, adapted from his figure 7.

The use of a thermal electron distribution introduces the minimum number of free parameters, and so leads to the simplest models. However, as Brinkmann[7] points out, the distribution might be truncated to soma extent. If this is the case, it will reduce the cooling, (essentially by mimicking a lower temperature) and so move the boundary line up.

\section{References}

1 R. Svensson, Ap.J., 258, 335 (1982).

2 A.P. Lightman, (these proceedings).

3 S. Stepney, MNRAS, 202, 467 (1983).

4 P.W. Guilbert, MNRAS, 197, 451 (1981).

5 S. Stepney, \& P.W. Guilbert, MNRAS, (in press).

6 R. Svensson, preprint (1982).

7 W. Brinkmann, (these proceedings). 